\title{
Artigo/Article
}

\section{Fauna de flebotomíneos (Diptera: Psychodidae) de uma reserva de campina no Estado do Amazonas, e sua importância epidemiológica}

\author{
Phlebotomine fauna (Diptera: Psychodidae) in a campina reserve in the State of Amazonas, \\ and its epidemiological importance
}

\author{
Plínio Eudson Santos da Silva ${ }^{1}$, Rui Alves de Freitas ${ }^{1}$, Dílvia Ferreira da Silva ${ }^{2}$ e Ronildo Baiatone Alencar ${ }^{1}$
}

\begin{abstract}
RESUMO
Introdução: No Brasil, a maior diversidade de flebotomíneos é encontrada na Região Amazônica, principalmente em floresta primária de terra-firme. No entanto, sua fauna ainda é pouco conhecida em outros ecossistemas desta região. Métodos: A fim de conhecer a fauna de flebotomíneos de outro ecossistema amazônico, coletas com armadilhas de luz CDC foram realizadas em uma área de campina/campinarana na zona periurbana da Cidade de Manaus. Resultados: Foram capturados 814 flebotomíneos de 26 espécies, sendo as mais abundantes: Lutzomyia flaviscutellata (64,5\%); Lutzomyia georgii (14\%); Lutzomyia olmeca nociva (8,1\%); Lutzomyia furcata (3,2\%); Lutzomyia monstruosa (1,3\%); Lutzomyia umbratilis $(1,1 \%)$ outros $(7,8 \%)$.Conclusões: Campina/campinarana revelou-se de grande importância epidemiológica devido a elevada abundância de Lutzomyia flaviscutellata e Lutzomyia olmeca nociva, ambas vetoras de Leishmania (Leishmania) amazonensis. Com base nestas informações, será possível traçar metas para estudos parasitológicos e conscientizar pesquisadores e estudantes, que mantém constante exposição nesta área realizando trabalhos de pesquisa, sobre o possível risco de contaminação por Leishmania.
\end{abstract}

Palavras-chaves: Lutzomyia. Epidemiologia. Campina. Amazônia.

\begin{abstract}
Introduction: In Brazil, the largest diversity of phlebotomines is found in the Amazon region, particularly in undisturbed terra-firma forest. However, the phlebotomine fauna in other ecosystems of this region is still not well known. Methods: In order to investigate the phlebotomine fauna of another Amazon ecosystem, collections were made using CDClight traps in the campina/campinarana ecosystems in the periurban zone of the city of Manaus. Results: Eight hundred and fourteen phlebotomines of 26 species were captured, of which the most abundant were: Lutzomyia flaviscutellata (64.5\%); Lutzomyia georgii (14\%); Lutzomyia olmeca nociva (8.1\%); Lutzomyia furcata (3.2\%); Lutzomyia monstruosa (1.3\%); Lutzomyia umbratilis (1.1\%); and others (7.8\%). Conclusions: The campina/campinarana ecosystems showed great epidemiological importance because of the high abundance of Lutzomyia flaviscutellata and Lutzomyia olmeca nociva, which are both vectors of Leishmania (Leishmania) amazonensis. Based on this information, it will be possible to outline goals for parasitological studies and raise awareness among researchers and students who are constantly exposed in these areas while conducting research work, regarding the possible risk of contamination by Leishmania.
\end{abstract}

Key-words: Lutzomyia. Epidemiology. Campina. Amazon region.

1. Coordenação de Pesquisas em Ciências da Saúde, Instituto Nacional de Pesquisas da Amazônia, Manaus, AM. 2. Laboratório de Biologia dos Insetos da Universidade Federal Rural de Pernambuco, Recife, PE.

Endereço para correspondência: Sr. Ronildo Baiatone Alencar. CPEn/INPA. Av. André Araújo 2936, Aleixo, 69060-001 Manaus, AM.

Tel: 55092 3643-3021

e-mail: ronildo@inpa.gov.br

Recebido para publicação em 10/08/2009

Aceito em 09/12/2009

\section{INTRODUÇÃO}

Os flebotomíneos constituem um grupo bem definido de insetos, provavelmente de origem monofilética ${ }^{1}$. Na Região Neotropical, estão representados pelos gêneros Lutzomyia, Brumptomyia e Warileya. No gênero Lutzomyia, são conhecidas cerca de 400 espécies $^{2}$, das quais aproximadamente 30 têm sido incriminadas como vetores comprovados ou suspeitos na transmissão de leishmânias ao homem ${ }^{3-6}$.

No Brasil, a área de floresta tropical, como a região amazônica, em virtude da maior variedade de nichos ecológicos, apresenta uma alta diversidade de flebotomíneos ${ }^{7}$. Entretanto, nesta região, a maioria dos estudos está voltada para espécies vetoras de Leishmania ${ }^{5}$ ou para levantamento de fauna em pequenas áreas de floresta primária ${ }^{8,9}$. Por outro lado, pouco se conhece sobre a fauna destes insetos em outros ecossistemas que compõem a região amazônica, como as campinas e campinaranas, refúgios montanhosos, savanas amazônicas, matas de igapó entre outros.

O conhecimento da distribuição e ecologia dos flebotomíneos é de fundamental importância para a vigilância epidemiológica das leishmanioses. $\mathrm{O}$ presente estudo teve como objetivo investigar a fauna flebotomínica de um ecossistema de campina/ campinarana localizado na área periurbana da Cidade de Manaus, com o intuito de contribuir para a caracterização da diversidade e distribuição destes insetos na Amazônia, enfatizando aqueles de interesse médico, para elucidar possíveis ciclos de transmissão de leishmânias nesses ecossistemas.

\section{MÉTODOS}

O estudo foi realizado na Reserva Biológica da Campina (6000’00” W; 2030'00” S) do Instituto Nacional de Pesquisas da Amazônia, localizada ao norte da Cidade de Manaus, no Km 45 da Rodovia BR-174 que liga Manaus, AM a Boa Vista, RR. 
A campina amazônica é definida por uma vegetação de porte baixo e aberta com característica xeromórfica e que cresce em solo arenoso lixiviado. O ecótono entre campina e floresta é denominado campinarana, onde o dossel é mais fechado que na campina. As campinas/campinaranas florescem em um ambiente inóspito e seletivo onde a pressão ecológica é intensa, e a drenagem ocorre rapidamente levando os nutrientes do solo. Devido a tais condições, a vegetação tende a desenvolver mecanismos adaptativos, que são claramente visíveis em seu aspecto fisionômico. Apresentam biomassa reduzida, baixa diversidade, escleromorfismo acentuado, e alto grau de endemismo ${ }^{10-12}$.

As coletas foram realizadas durante o período de setembro de 2005 a janeiro de 2006. Foram utilizadas 20 armadilhas luminosas do tipo CDC (Hausher's Machine Works, New Jersey, Estados Unidos) modificadas com potes coletores, contendo álcool a 70\%. As armadilhas foram instaladas por três noites consecutivas de cada mês, sempre nos mesmos locais, a 1 metro do nível do chão, e distante $10 \mathrm{~m}$ uma da outra em um transecto de $190 \mathrm{~m}$ na área de campina/campinarana. Todas as armadilhas eram instaladas as 18:00h e recolhidas as $06: 00 \mathrm{~h}$. Os flebotomíneos coletados foram identificados de acordo com o catálogo de Young e Duncan ${ }^{2}$ e descrições originais.

\section{RESULTADOS}

As capturas totalizaram 814 flebotomíneos, distribuídos em 26 espécies do gênero Lutzomyia (Tabela 1), oito subgêneros (Psychodopygus, Nyssomyia, Evandromyia, Psathyromyia, Viannamyia, Trichophoromyia, Sciopemyia e Trichopygomyia) e três grupos de espécies (aragaoi, migonei e oswaldoi).

As seis espécies mais representativas somaram $92,2 \%$ dos espécimes coletados, com destaque para Lutzomyia flaviscutellata, que totalizou $64,5 \%$. Nove espécies foram representadas por apenas um indivíduo cada (Tabela 1). Os subgêneros Psychodopygus, Nyssomyia e Evandromyia foram os mais representativos com 6, 5 e 4 espécies, repectivamente. Destes, o segundo somou $73 \%$ dos flebotomíneos coletados, a maioria sendo representado por Lutzomyia flaviscutellata.

O número de flebotomíneos variou mensalmente, influenciado principalmente pelo número de indivíduos das quatro primeiras espécies mais representativas, em particular de Lutzomyia flaviscutellata. Os meses de novembro, dezembro e janeiro foram os mais produtivos com 270, 304 e 140 indivíduos, respectivamente, enquanto o mês de setembro foi o de menor produção com 13 indivíduos coletados.

TABELA 1 - Composição e distribuição mensal das espécies de flebotomíneos capturadas com armadilhas luminosas (CDC) em uma área de campina, Manaus-AM, no período de setembro de 2005 a janeiro de 2006.

\begin{tabular}{|c|c|c|c|c|c|c|c|c|c|}
\hline \multirow[b]{2}{*}{ Espécie } & \multicolumn{5}{|c|}{ Meses } & \multicolumn{2}{|c|}{ Sexo } & \multirow[b]{2}{*}{ Total } & \multirow[b]{2}{*}{$\%$} \\
\hline & set & out & nov & dez & jan & M & $\mathrm{F}$ & & \\
\hline Lutzomyia flaviscutellata* & 4 & 51 & 150 & 224 & 96 & 195 & 330 & 525 & 64,5 \\
\hline Lutzomyia georgii & - & 16 & 62 & 29 & 7 & - & 114 & 114 & 14,0 \\
\hline Lutzomyia olmeca nociva* & 2 & 5 & 15 & 28 & 16 & 21 & 45 & 66 & 8,1 \\
\hline Lutzomyia furcata* & 1 & 1 & 16 & 1 & 7 & 3 & 23 & 26 & 3,2 \\
\hline Lutzomyia monstruosa & 1 & 4 & 3 & 2 & - & 2 & 8 & 10 & 1,3 \\
\hline Lutzomyia umbratilis* & - & - & 5 & 3 & 1 & 1 & 8 & 9 & 1,1 \\
\hline Lutzomyia tuberculata* & 2 & 2 & - & - & 3 & - & 7 & 7 & 0,9 \\
\hline Lutzomyia sordellii & - & - & 3 & 2 & 2 & 2 & 5 & 7 & 0,9 \\
\hline Lutzomyia paraensis* & - & - & - & 3 & 4 & 2 & 5 & 7 & 0,9 \\
\hline Lutzomyia ayrozai* & - & 1 & 1 & 3 & 1 & 1 & 5 & 6 & 0,7 \\
\hline Lutzomyia ratcliffei & - & - & 3 & 2 & 1 & 4 & 2 & 6 & 0,7 \\
\hline Lutzomyia inpai & - & - & 5 & 1 & - & 4 & 2 & 6 & 0,7 \\
\hline Lutzomyia rorotaensis & - & 4 & 1 & - & - & - & 5 & 5 & 0,6 \\
\hline Lutzomyia aragaoi & - & 1 & 2 & 1 & 1 & - & 5 & 5 & 0,6 \\
\hline Lutzomyia anduzei* & 1 & - & - & 1 & - & 1 & 1 & 2 & 0,3 \\
\hline Lutzomyia (Psathyromyia) sp & 2 & - & - & - & - & 2 & 2 & 0,3 & \\
\hline Lutzomyia sq. squamiventris* & - & - & - & 2 & - & 1 & 1 & 2 & 0,3 \\
\hline Lutzomyia antunesi & 1 & - & - & - & - & - & 1 & 1 & 0,1 \\
\hline Lutzomyia tarapacaensis & 1 & - & - & - & - & - & 1 & 1 & 0,1 \\
\hline Lutzomyia scaffi & - & - & 1 & - & - & - & 1 & 1 & 0,1 \\
\hline Lutzomyia bispinosa & - & - & 1 & - & - & - & 1 & 1 & 0,1 \\
\hline Lutzomyia ubiquitalis* & - & - & 1 & - & - & 1 & - & 1 & 0,1 \\
\hline Lutzomyia claustrei & - & - & 1 & - & - & 1 & - & 1 & 0,1 \\
\hline Lutzomyia eurypyga & - & - & - & 1 & - & - & 1 & 1 & 0,1 \\
\hline Lutzomyia davisi & - & - & - & - & 1 & - & 1 & 1 & 0,1 \\
\hline Lutzomyia sp de Baduel & - & - & - & 1 & - & - & 1 & 1 & 0,1 \\
\hline Total & 13 & 87 & 270 & 304 & 140 & 239 & 575 & 814 & 100,0 \\
\hline
\end{tabular}

*Espécies incriminadas como vetores comprovados ou suspeitos na transmissão de leishmanias ao homem em diferentes regiões do Brasil ${ }^{36}$. 


\section{DISCUSSÃO}

A grande diversidade e abundância de flebotomíneos, principalmente do gênero Lutzomyia, encontrada em matas de zonas de baixas latitudes da América Central e do Sul, permite em um único hectare de floresta o registro de até 50 espécies ${ }^{13}$.

O número de espécies encontradas no presente estudo encontrase abaixo do que normalmente é registrado para florestas primárias na região amazônica. Comparativamente, Dias-Lima et a ${ }^{8}$, em seis meses de coleta com armadilha CDC em uma floreta primária adjacente ao local do presente estudo, registraram 7.409 flebotomíneos distribuídos em 41 espécies. Esta diferença pode ser reflexo das diferentes condições climáticas e microclimáticas específicas destes locais. De acordo com Aguiar e Medeiros ${ }^{7}$, os abrigos dos flebotomíneos adultos variam de acordo com o microhabitat, estação do ano, umidade relativa do ar e também com as espécies. Estes, em geral se abrigam em locais relativamente úmidos, com matéria orgânica em decomposição abundante e pouca luminosidade onde possam proteger-se de mudanças climáticas bruscas.

De fato, estas condições não são inteiramente encontradas em áreas de campina/campinarana, em virtude principalmente, da intensa penetração de luz ocasionada pela vegetação arbustiva e esparsa em relação à floresta primária. Além disso, o terreno arenoso desse ecossistema permite uma rápida drenagem da água na superfície do solo. De acordo com Ward et $\mathrm{al}^{14}$, a densidade populacional de certas espécies silvestres depende mais da quantidade de água das chuvas retida pelo solo, que do próprio volume pluviométrico.

Entretanto, em florestas tropicais, a densidade de flebotomíneos parece estar diretamente relacionada com a precipitação pluviométrica, sendo mais abundantes no período de maior precipitação ${ }^{15,16}$. Na Amazônia, o período de chuvas ou forte atividade convectiva é compreendido entre novembro e março, enquanto o período de seca (sem grande atividade convectiva) é entre os meses de maio e setembro. Os meses de abril e outubro são meses de transição entre um regime e outro ${ }^{17}$.

Os resultados obtidos na campina parecem estar em consonância com resultados do ambiente de floresta tropical, tendo em vista que os períodos de maior e menor captura ocorreram nos meses de maior e menor precipitação para a região, respectivamente. Obviamente, o período de estudo foi relativamente curto para permitir uma análise mais conclusiva a respeito dessa possível flutuação sazonal.

Não foi registrada qualquer espécie de flebotomíneo endêmica para a área de campina neste estudo, visto que todas as espécies registradas são também normalmente encontradas em floretas primárias na região amazônica ${ }^{8,9,18}$. Contudo, verificaram-se grandes diferenças quanto à abundância relativa de algumas espécies entre estes dois ecossistemas. Lutzomyia umbratilis e Lutzomyia anduzei, por exemplo, que normalmente são encontradas em grandes proporções em coletas com armadilhas luminosas (CDC) nas matas próximas a campina ${ }^{8,9}$, contribuíram, conjuntamente, com menos de 1,5\% dos espécimes capturados (Tabela 1). Estas duas espécies são de grande interesse epidemiológico na região amazônica, pois são vetores potenciais de Leishmania (Viannia) guyannensis, que adquirem de mamíferos arborícolas como bicho-preguiça (Choloepus didactylus), tamanduá (Tamandua tetradactyla) e marsupiais do gênero Didelphis naturalmente infectados ${ }^{19-21}$. A baixa densidade de Lutzomyia umbratilis e Lutzomyia anduzei no ecossistema de campina/campinarana pode ser explicada pela ausência de abrigos naturais (tronco de árvores) e conseqüentemente pela ausência de mamíferos que habitualmente freqüentam o dossel de mata alta e que servem como recursos de fonte sanguínea pelas fêmeas dessas espécies.

Por outro lado, Lutzomyia flaviscutellata, que na floresta primária é raramente encontrada sobre troncos de árvores ou coletada em armadilha de luz (CDC $)^{22}$, na campina, foi a espécie predominante. Shaw e Lainson ${ }^{23}$ coletaram esta espécie em grande quantidade em ambiente de floresta primária, porém utilizando armadilha Disney em nível do chão.

Assim, como Lutzomyia umbratilise Lutzomyia anduzei, Lutzomyia flaviscutellata também é de grande importância epidemiológica, pois é incriminada como vetor de Leishmania (Leishmania) amazonensis ${ }^{23,24}$. É predominantemente encontrada picando pequenos roedores em nível do chão, onde sua densidade é maior, e apresenta pouca tendência para picar o homem ${ }^{25}$. Todavia, infecções ocasionais neste último podem ocorrer, principalmente, porque as chances de se infectar com Leishmania amazonensis, aumentam com o aumento da densidade do vetor ${ }^{26}$. Nesse sentido, as pessoas que normalmente freqüentam o ambiente de campina/campinarana correm grande risco de contaminação por Leishmania. Além disso, também foi encontrada com relativa freqüência Lutzomyia olmeca nociva, outro táxon vetor de Leishmania amazonensis. Embora a presença e densidade desses vetores exerçam papel fundamental na transmissão de leishmânia para o homem, estudos mais aprofundados, que possam investigar a presença e a taxa de infecção por Leishmania amazonensis nestas espécies, são necessários para avaliar o verdadeiro risco a que estão submetidos pesquisadores, técnicos e estudantes que freqüentemente utilizam as campinas e campinaranas em atividades de pesquisas.

A segunda espécie mais abundante foi Lutzomyia georgii (Tabela 1). Descrita somente em janeiro de $2002^{27}$, sua presença não é registrada em trabalhos anteriores a esta data, devido ter sido identificada como Lutzomyia begonae ${ }^{28}$ e, posteriormente, tratada como variante de Lutzomyia infraspinosa ${ }^{2}$. Apesar da sua presença marcante na campina/campinarana, Lutzomyia georgii não é incriminada como vetora de Leishmania, assim como Lutzomyia monstruosa, outra espécie bem representada neste estudo.

Lutzomyia furcata, Lutzomyia tuberculata, Lutzomyia paraensis, Lutzomyia ayrozai, Lutzomyia squamiventris squamiventris e Lutzomyia ubiquitalis, embora pouco representadas na campina/campinarana, são incriminadas como vetores comprovados ou suspeitos na transmissão de leishmânias ao homem ${ }^{3-6}$. Dessas, Lutzomyia furcata foi a mais representativa com 26 indivíduos, somando 3,2\% do total, e assim como Lutzomyia flaviscutellata e Lutzomyia olmeca nociva foi registrada em todos os meses de coleta (Tabela 1), representando assim mais uma espécie de importância epidemiológica para o ambiente de campina/campinarana.

Em geral, a maioria das espécies foi representada por menos de dez indivíduos (Tabela 1). Tal resultado sugere um alto grau de rejeição desses flebotomíneos pelo ambiente de campina/campinarana, devido, provavelmente, às condições climáticas adversas desse ecossistema ou pela falta de fontes sanguíneas e abrigos diurnos para os adultos. Entretanto, estudos mais prolongados e que contemplem outras áreas de campinas e campinaranas, bem como o uso de outras técnicas de capturas, além de estudos parasitológicos, são necessários para melhor caracterizar e avaliar a fauna flebotomínica nesses ecossistemas, quanto a sua real diversidade e importância epidemiológica. 


\section{CONFLITO DE INTERESSE}

Os autores declaram não haver nenhum tipo de conflito de interesse.

\section{AGRADECIMENTOS}

Aos técnicos Francisco Lima Santos e Lourival Maciel Castro, pelas coletas em campo. Ao Dr. Márcio Luís Leitão Barbosa, pelas críticas e sugestões na redação do manuscrito. A Dra. Vera Margarete Scarpassa, Gersonval Leandro Silva Monte e Rafael Augusto Pinheiro de Freitas Silva, pela revisão do abstract.

\section{REFERÊNCIAS}

1. Kettle DS. Medical and Veterinary Entomology. $2^{\text {nd }}$ edition, CAB International Publishing, Wallingford; 1995.

2. Young DG, Duncan MA. Guide to the identification and Geographic Distribution of Lutzomyia Sand Flies in Mexico, the West Indies, Central and South America (Diptera: Psychodidae). Associated Publishers, Gainesville, Florida, USA; 1994.

3. Braga RR, Lainson R, Ishikawa EA, Shaw JJ. Leishmania (Viannia) utingensis $\mathrm{n}$. sp, a parasite from the sandfly Lutzomyia (Viannamyia) tuberculata in Amazonian Brazil. Parasite 2003; 10: 111-118.

4. Killick-Kendrick R. Phlebotomine vectors of leishmaniasis: a review. Medical and Veterinary Entomology 1990; 4: 1-24.

5. Lainson R, Shaw JJ. Leishmania (Viannia) naiffi sp.n. a parasite of the armadillo, Dasypus novencinctus (L.) in Amazonian Brazil. Ann Parasitol Hum Comp 1989; 64: 3-9.

6. Williams P. Psychodidae. In: Neves DP, Melo AL, Genaro O, Linardi PM (eds) Parasitologia Humana; 2003. p. 311-319.

7. Aguiar GM, Medeiros WM. Distribuição regional de habitats das espécies de flebotomíneos do Brasil. In: Rangel EF, Lainson R (eds). Flebotomíneos do Brasil; 2003. p. 207-256.

8. Dias-Lima AG, Castellón EG, Sherlock I. Flebotomíneos (Diptera: Psychodidae) de uma floresta primária de terra firme da Estação Experimental de Silvicultura Tropical, Estado do Amazonas, Brasil. Acta Amazonica 2003; 33: 303-316.

9. Feitosa MAC, Castellón EG. Fauna de flebotomíneos (Diptera: Psychodidae) em fragmentos de floresta ao redor de conjuntos habitacionais na cidade de Manaus, Amazonas, Brasil. I. Estratificação Vertical. Acta Amazônica 2006; 36: 539-548.

10. Anderson AB, Prance GT, Albuquerque BWP. Estudo sobre a vegetação das campinas amazônicas - III A vegetação lenhosa da Campina da reserva Biológica INPA - SUFRAMA. Acta Amazonica 1975; 5: 225-246.

11. Pires JM, Prance GT. The vegetation types of the Brazilian Amazon. In: Prance GT, Lovejoy TE (eds). Key Environments: Amazonia, Pergamon Press, Oxford; 1985. p. 109-145.

12. Prance GT. Islands in Amazonia. Phil. Trans. R. Soc. B 1975; 351: 823-833.

13. Young DG, Arias JR. Flebotomíneos vectores de leishmaniasis en las Americas. Organização Pan-Americana da Saúde, caderno técnico (3); 1992.

14. Ward RD, Shaw JJ, Lainson R, Fraiha H. Leishmaniasis in Brazil: VIII. Observations on the phlebotomine fauna of an area highly endemic for cutaneous leishmaniasis, in the Serra dos Carajás, Pará State. Trans R Soc Trop Med Hyg 1973; 67: 174-183.

15. Arias JR, Freitas RA. On the vectors of cutaneous leishmaniasis in the Central Amazon of Brazil. 4. Sand fly emergence from a "terra firme" forest floor. Acta Amazonica 1982; 12: 609-611.

16. Ximenes MFFM, Castellón EG, Souza MF, Menezes AAL, Queiroz JW, Silva VPM, Jerônimo SMB. The effect of abiotic factors on seasonal population dynamics of Lutzomyia longipalpis (Diptera: Psychodidae) in northeastern Brazil. J Med Entomol 2006; 43: 990-995.

17. Fisch G, Marengo JA, Nobre CA. Clima da Amazônia. Boletim do Climanálise Especial - Comemoração dos 10 anos, 1996. Disponível em: http://www.cptec. inpe.br/products/climanalise/cliesp10a. Acesso em: 04 de Maio de 2009.

18. Silva DF, Freitas RA, Franco AMR. Diversidade e abundância de flebotomíneos do gênero Lutzomyia (Diptera: Psychodidae) em Áreas de Mata do Nordeste de Manacapuru, AM. Neotro Entomo 2007; 36: 138-144.

19. Arias JR, Naiff RD. The principal reservoir host of cutaneous leishmaniasis in the urban area of Manaus, Central Amazon of Brazil. Mem Inst Oswaldo Cruz 1981; 76: 279-286.

20. Gentile B, Le Pont F, Pajot FX, Bernard R. Dermal leishmaniasis in French Guiana: the sloth (Choloepus didactylus) as a reservoir host. Trans R Soc Trop Med Hyg 1981; 75: 612-613.

21. Lainson R, Shaw JJ, Povoa M. The importance of edentates sloths and anteaters as primary reservoirs of Leishmania braziliensis guyanensis, causative agent of "Pian-bois" in Northern Brazil. Trans R Soc Trop Med Hyg 1981; 75: 611-612.

22. Shaw JJ, Lainson R. Leishmaniasis in Brazil: VI. Observations on the seasonal variations of Lutzomyia flaviscutellata in different types of forest and its relationship to enzootic rodent leishmaniasis (Leishmania mexicana amazonensis). Transactions of the Royal Society of Tropical Medicine and Hygiene 1972; 66: 709-717.

23. Shaw JJ, Lainson R. Leishmaniasis in Brazil: II Observsations on enzootic rodent leishmaniasis in the lower amazon region - The feeding habitats of the vector, Lutzomyia flaviscutellata in reference to man, rodents and other animals. Trans R Soc Trop Med Hyg 1968; 62: 396-405.

24. Lainson R, Shaw JJ. Leishmaniasis in Brazil. I - Observations on enzootic rodent leishmaniasis - Incrimination of Lutzomyia flaviscutellata (Mangabeira) as the vector in the lower Amazonian basin. Trans R Soc Trop Med Hyg 1968; 62: 385-395.

25. Shaw JJ, Lainson R. Leishmaniasis in Brazil: III. Cutaneous leishmaniasis in an Opossum, Marmosa murina (Marsupialia, Didelphidae) from the lower amazon region. Trans R Soc Trop Med Hyg 1969; 63: 738-740.

26. Shaw JJ, Lainson R, Ward RD. Leishmaniasis in Brazil: VII. Further observations on the feeding habitats of Lutzomyia flaviscutellata (Mangabeira) with particular reference to its biting habits at different heights. Trans $\mathrm{R}$ Soc Trop Med Hyg 1972; 66: 718-723.

27. Freitas RA, Barrett TV. Description of Lutzomyia (Evandromyia) georgii n. sp. and a Synopsis of the Series infraspinosa (Diptera: Psychodidae). Mem Inst Oswaldo Cruz 2002; 97: 239-245.

28. Ortiz I, Torrez JR. Phlebotomus begonae nov. sp. flebótomo del subgênero Evandromyia Mangabeira, 1941 em La région nor-occidental Amazónica Venezoelana (Diptera: Psychodidae). Rev Inst Nac de Higiene Caracas 1975; 8: 101-105. 